\title{
Die Beamtenversorgung zwischen Modernisierung und Sparzwang
}

Seit der Dienstrechtsreform 1997 soll sich besondere Leistung auch für Beamte finanziell lohnen. Allerdings haben die neuen leistungsabhängigen Besoldungselemente bisher keinen Einfluss auf die Versorgungsansprüche aus der Beamtenversorgung. Vielmehr bleiben sie bei der Ruhegehaltsberechnung unberücksichtigt. Hier stellt sich die Frage, warum die auf Leistungsanreize abzielenden Reformmaßnahmen nicht auch auf das Alterssicherungssystem der Beamten übertragen wurden. ${ }^{1}$

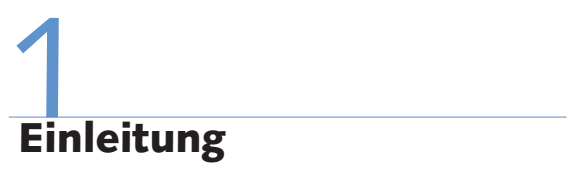

Vor gut einem Jahrzehnt hat der Gesetzgeber begonnen, den öffentlichen Dienst in Deutschland mit dem Ziel einer wettbewerbsfähigen und leistungsorientierten Dienstleistungskultur umzugestalten. Die Ursachen für diese „Modernisierung“ waren unter anderem die angespannte Haushaltslage, fehlendes betriebswirtschaftliches Denken, Mängel in der Personalwirtschaft im Sinne ungenutzter Leistungspotenziale, Unzufriedenheit und Motivationsdefizite bei den Bediensteten sowie ein Vergütungssystem, das im Gegensatz zur Privatwirtschaft kaum eine Entlohnung der individuellen Leistung des Einzelnen zuließ (Becker et al. 2007, S. 9ff.). Auch das öffentliche Dienstrecht für Beamte wurde im Rahmen der Verwaltungsmodernisierung, hier zu verstehen als die Stärkung des Leistungsprinzips durch leistungsorientierte Bezahlung und Lockerung des Laufbahnrechts (Bull 2008, S. 230f.), reformiert. Heute gilt auf Bundesebene ein Besoldungsrecht, welches das Leistungsprinzip durch leistungsorientierte Besoldungselemente unterstützen will.

Mit dem am 11. Februar 2009 in Kraft getretenen Dienstrechtsneuordnungsgesetz (DNeuG, BGBl. I S. 160, BT-Drs. 16/ 7076) hat der Gesetzgeber noch einmal die Absicht betont, für den Bund ein Beamtenrecht zu schaffen, das die Leistungsorientierung im öffentlichen Dienst fördert. In diesem Artikel wird die Frage aufgeworfen, warum Elemente der leistungsorientierten Besoldung bisher keine Berücksichtigung bei der Ruhegehaltsberechnung der Beamtenversorgung finden. Zur Beantwortung dieser Fragestellung werden zunächst die
Strukturprinzipien der Beamtenversorgung vorgestellt (Abschnitt 2), bevor die leistungsorientierten Elemente der Besoldung und ihre Verbindung zur Beamtenversorgung erörtert werden (Abschnitt 3). Abschnitt 4 wendet sich den Finanzierungsproblemen der Beamtenversorgung zu, aus denen der „Sparzwang" der Gebietskörperschaften resultiert. In Abschnitt 5 wird schließlich anhand erfolgter Reformmaßnahmen diskutiert, ob die Beamtenversorgung in den letzten Jahren im oben genannten Sinn modernisiert oder ob lediglich am Versorgungsniveau gespart wurde. Der Artikel schließt mit einem Ausblick (Abschnitt 6), der die Auswirkungen auf den öffentlichen Dienst als Arbeitgeber thematisiert.

\section{Strukturprinzipien der Beamtenversorgung}

Anders als die gesetzliche Rentenversicherung wird die Altersversorgung der Beamten, die insbesondere durch das Beamtenversorgungsgesetz (BeamtVG) geregelt wird, unmittelbar vom Dienstherrn selbst erbracht. Der Beamte entrichtet keine (direkten) eigenen Beiträge zu seiner Alterssicherung, sondern seine Versorgung stellt einen öffentlich-rechtlichen, gesetzlich geregelten Unterhaltsanspruch gegen den Dienstherrn dar (Murmann 1991, S. 232). Verfassungsrechtlich verankert sind die Grundlagen der Beamtenversorgung in Art. 33 Abs. 5 GG. Danach ,ist das Recht des öffentlichen Dienstes unter Berücksichtigung der hergebrachten Grundsätze des Berufsbeamtentums zu regeln und fortzuentwickeln“. Im versorgungsrechtlichen Sinne zählt zu diesen Grundsätzen neben dem Lebenszeitprinzip sowie der Fürsorgepflicht des Dienstherrn vor allem das Alimentationsprinzip. Die Alimentation, d.h. die staatliche Unterhaltsgewährung für Beamte, hat ihre Wurzeln im 18. Jahrhundert und fußt auf der tradierten Auffassung, dass eine geordnete Staatsverwaltung und eine Verpflichtung auf das Staats- und Gemeinwohl nur dann sichergestellt ist, wenn die verantwortlichen Amtsträger auf Lebenszeit wirtschaftlich abgesichert sind. Die Funktion der Beamtenversorgung besteht demnach darin, die angemessene Lebenshaltung des Beamten entsprechend dem zuletzt bekleideten Amt für die Zeit nach Ausscheiden aus dem öffentlichen Dienst zu garantieren (BullKommission 2003, S. 45).

Kernbestandteil der Beamtenversorgung ist das Ruhegehalt, welches ab Erreichen bestimmter Altersgrenzen oder beim Eintreten von Dienstunfähigkeit bezogen wird. Aufgrund der Neuregelungen des

Der vorliegende Beitrag folgt einem Call for Papers der WSI-Mitteilungen zum Thema "Der Staat als Arbeitgeber ${ }^{\prime}$, der unter www.boeckler.de/pdf/ wsimit_2009_call_for_paper_ag_staat.pdf abgerufen werden kann.

Melanie Funke, Juristin und Verwaltungswissenschaftlerin, ist Forschungsreferentin am Deutschen Forschungsinstitut für öffentliche Verwaltung in Speyer. Arbeitsschwerpunkte: Finanzierung der Beamtenversorgung, öffentliches Dienstrecht. e-mail: funke@foev-speyer.de Steffen Walther, Volkswirt und Verwaltungswirt (FH), ist Forschungsreferent am Deutschen Forschungsinstitut für öffentliche Verwaltung in Speyer. Arbeitsschwerpunkt: Finanzierung der Beamtenversorgung, Alterssicherung.

e-mail:walther@foev-speyer.de 
DNeuG wird - wie in der gesetzlichen Rentenversicherung auch - die Regelaltersgrenze ab 2012 schrittweise von 65 auf 67 Jahre angehoben. ${ }^{2}$ Die Antragsaltersgrenze bleibt bei 63 Jahren, jedoch erhöht sich der maximale Versorgungsabschlag von $10,8 \%$ auf $14,4 \%$. Kann der Beamte 45 Dienstjahre nachweisen, so ist gleichwohl ein abschlagsfreier Eintritt in den Ruhestand mit 65 Jahren möglich.

Berechnet wird das Ruhegehalt seit dem 1. Januar 1992 nach folgender Ruhegehaltsformel (vgl. \$4 Abs. 3 BeamtVG):

Ruhegehalt $($ Pension $)=$ Ruhegehaltsfähige Dienstjahre $\times$ Steigerungssatz pro Jahr in \% $\times$ Ruhegehaltsfähige Dienstbezüge

$\mathrm{Zu}$ den ruhegehaltsfähigen Dienstjahren zählen die Dienst-, Ausbildungs- und Zurechnungszeiten, eventuelle Beschäftigungszeiten als Angestellter im öffentlichen Dienst sowie Wehrdienst und vergleichbare Zeiten ( $\$ \$ 6 f f$. BeamtVG). Der maximale Höchstruhegehaltssatz betrug im Jahr 2009 72,56 \%3 (zukünftig 71,75\%) und wird nach 40 ruhegehaltsfähigen Dienstjahren erreicht. Zur Bestimmung des Ruhegehaltssatzes wird die Anzahl dieser Jahre mit dem Steigerungssatz von $1,81402 \%$ (zukünftig 1,79375\%) multipliziert. Die ruhegehaltsfähigen Dienstbezüge setzen sich aus dem Grundgehalt des letzten Amtes, welches der Beamte mindestens zwei Jahre lang ausgeübt haben muss (BVerfG, Beschl. v. 20.3.2007 - 2 BvL 11/04), dem Familienzuschlag der Stufe 1 sowie sonstigen Dienstbezügen, die im Besoldungsrecht ausdrücklich als ruhegehaltsfähig ausgewiesen sind, zusammen.

\section{3 \\ Ruhegehalt und leistungs- orientierte Besoldung}

Über die Höhe der ruhegehaltsfähigen Dienstbezüge hat die Ausgestaltung der Beamtenbesoldung einen direkten Einfluss auf die Höhe der Versorgungsbezüge. Die Besoldung der Bundesbeamten wird durch das Beamtenbesoldungsgesetz (BBesG) geregelt. ${ }^{4}$ Maßgebend für die Höhe der Besoldung ist im Sinne des Alimentationsprinzips die Wertigkeit des ausgeübten Amtes. Diese bestimmt sich anhand innerdienstlicher, unmittelbar auf das Amt bezogener Kriterien wie der fachlichen Qua- lifikation („Leistungsprinzip“), der damit einhergehenden Eingruppierung sowie dem Dienstrang („Laufbahnprinzip“) und der mit dem Amt verbundenen Verantwortung (BVerfGE 114, 258 287f.; Lindner 2007, S. 222).

\subsection{DIE EINFÜHRUNG VON LEISTUNGSELEMENTEN IN DAS VERGÜTUNGSSYSTEM}

Im Rahmen der Umgestaltung des öffentlichen Sektors wollte der Gesetzgeber mit dem am 1. Juli 1997 in Kraft getretenen Dienstrechtsreformgesetz die Leistungselemente im Besoldungssystem stärken und es attraktiver sowie flexibler gestalten (Biermann et al. 2005, S. 114f.). Die Reformen folgten hierbei der Grundidee der Verwaltungsmodernisierung, indem Konzepte des betriebswirtschaftlichen Managements auf die öffentliche Verwaltung übertragen werden sollten (Biermann et al. 2005, S. 27ff.; Lorse 2007, S. 24), d.h. das Verwaltungshandeln und die Aufgabenwahrnehmung sollten effizienter, effektiver und transparenter durchgeführt sowie qualitativ verbessert werden. Die Bezahlung sollte deshalb nicht mehr ausschließlich nach Alter, Familienstand und Kinderzahl erfolgen, sondern nach individueller Leistung und Berufserfahrung.

In Abkehr vom leistungsunabhängigen Bewährungs- und Zeitaufstieg („Besoldungsdienstalter" bzw. „Senioritätsprinzip“) wurden daher in das BBesG erstmals variable „Leistungskomponenten“ eingeführt. Die Grundgehaltstabelle wurde mit dem Anspruch, Leistungsaspekte in der Grundbesoldung zu verstärken, neu gefasst und anstelle eines leistungsunabhängigen zweijährigen Rhythmus bei „durchschnittlicher Leistung" auf einen Zwei-, Drei-, Vierjahresrhythmus umgestellt. Das Aufsteigen in die nächsthöhere Besoldungsstufe erfolgte somit nicht mehr allein durch Zeitablauf, sondern leistungsabhängig im Rahmen von „Leistungsstufen“.

Die Vergabe der Leistungsstufe hängt von einer "dauerhaft herausragenden“ Leistung ab. Bei unterdurchschnittlicher Leistung verbleibt der Beamte hingegen in der bisherigen Stufe, bis seine Leistung ein Aufsteigen in die nächsthöhere Stufe rechtfertigt ( $\$ 27$ Abs. 5, 7 BBesG). Die Vergabe einer Leistungsstufe durch Wechsel in die nächsthöhere Stufe stellt gleichwohl kein eigenständiges Bezahlungselement dar, sondern bedeutet automatisch die Erhö- hung des Grundgehaltes (Göser/Schlatmann 1998, S. 12). Da die erreichte Leistungsstufe das neue Grundgehalt darstellt, ist sie ruhegehaltsfähig.

Ebenfalls zur besonderen Honorierung „herausragender" Leistungen wurden als zusätzliche Leistungselemente in $\$ 42 \mathrm{a}$ BBesG „Leistungsprämien“ und „Leistungszulagen“ eingeführt, die das monatlich fixe Grundgehalt, dessen Höhe sich nach der Besoldungsgruppe des dem Beamten verliehenen Amtes bemisst, „aufstocken“. Bei der Leistungsprämie handelt es sich um eine Einmalzahlung, die bis zur Höhe des Anfangsgrundgehaltes der jeweiligen Besoldungsgruppe gewährt werden kann. Die Leistungszulage ist eine zeitlich befristete, widerrufliche, monatlich wiederkehrende Zahlung, deren Höhe monatlich $7 \%$ des Anfangsgrundgehaltes nicht übersteigen darf. Sowohl Leistungsprämie als auch Leistungszulage sind nicht ruhegehaltsfähig.

In Anbetracht der schwierigen öffentlichen Haushaltslage und unter dem Postulat der Personalausgabensenkung wurden die Leistungselemente kostenneutral eingeführt (Mühlenkamp 2008, S. 637). Der „Leistungstopf “ ist daher durch Absenkung der Grundbesoldung, insbesondere durch Kürzung bzw. Wegfall des Urlaubs- und Weihnachtsgelds, sowie aus auslaufenden Besitzständen aus Kinder- und Familienzuschlägen „erwirtschaftet“ worden (Battis 2005, S. 325). Außerdem dürfen pro Jahr maximal $15 \%$ der Beamten hinsichtlich der zu vergebenden Leistungsstufen und hinsichtlich der Leistungsprämien sowie -zulagen berücksichtigt werden.

Die beschränkende Regelung der Kostenneutralität und die 15-\%-Quotierung werden heftig kritisiert (Bull 2008, S. 231; Jörges-Süß 2006, S. 38f.; Mühlenkamp 2008), da aus ihnen große Umsetzungsund Vermittlungsprobleme der Reformen resultieren. Die Anreizwirkungen auf die Bediensteten fallen niedrig aus und es müssen negative Motivationseffekte für den Teil des Personals, der bei der Vergabe der Leis-

\footnotetext{
2 Auch für Polizei, Feuerwehr, Richter und Soldaten werden die besonderen Altersgrenzen schrittweise um zwei Jahre erhöht; ebenso für Schwerbehinderte.

3 6. Anpassungsfaktor für Bundesbeamte gemäß § 14 Abs. 1 i. V.m. § 69 e Abs. 3 BeamtVG.

4 Seit der am 1. September 2006 in Kraft getretenen Föderalismusreform I können die Länder eigene Regelungen hinsichtlich der Besoldung und Versorgung ihrer Beamten treffen.
} 
tungselemente nicht einbezogen wurde, befürchtet werden. Aus der Perspektive der Alterssicherung ist hingegen folgender Fakt herauszustellen: Eine Absenkung der ruhegehaltsfähigen Grundbesoldung bei gleichzeitiger Nichtberücksichtigung der Leistungszulagen und -prämien in der Ruhegehaltsberechnung ist letztendlich eine Minderung der Beamtenversorgungsleistungen. 5

\subsection{VON DER DIENSTRECHTSREFORM BIS ZUM DNeuG}

Die Änderungen des Dienstrechtsreformgesetzes sollten erst der Anfang eines fortlaufenden Reformprozesses sein. Im Jahr 2005 hatte die Bundesregierung den Entwurf eines Gesetzes zur Reform der Strukturen des öffentlichen Dienstrechts beschlossen (BT-Drs. 615/05; Battis 2005, S. 325ff.). Der Entwurf sah die Einführung eines umfassenderen leistungsbezogenen Bezahlungssystems vor. Im Unterschied zur Dienstrechtsreform von 1997 sollte sich die Einführung der Leistungsstufe individuell auf das Besoldungsniveau aller Beamten auswirken und die variable Leistungsbezahlung vollständig bei der Versorgung berücksichtigt werden (Schily et al. 2005; Lorse 2005, S. 445). Der Entwurf erledigte sich jedoch durch die Neuwahl des Bundestages im September 2005.

Das nun beschlossene DNeuG hat einen Großteil der Reformüberlegungen von 2005 nicht aufgegriffen, sondern mit einem Schritt zurück am Dienstrechtsreformgesetz von 1997 angeknüpft (Bolay 2007, S. 104; Drescher 2007, S 261f.; Battis 2005, S. 326). Das DNeuG setzt dabei weiterhin auf eine Abkehr vom Besoldungsdienstalter und dem Senioritätsprinzip, indem es den altersbezogenen Aufstieg in den Stufen abschafft. Im Zuge dessen erfolgte ab Juli 2009 in den Grundgehaltstabellen ein Wechsel zu (an beruflichen Dienstzeiten orientierten) Erfahrungsstufen. Die ursprünglich zwölf Grundgehaltstufen wurden einheitlich auf acht reduziert. Bestehende Leistungsbezahlungsinstrumente wie Leistungsstufe, Leistungszulage und Leistungsprämie wurden beibehalten. Des Weiteren wurde im DNeuG erstmals geregelt, dass das vorhandene Budget für die Leistungsbezahlung von $0,3 \%$ der jährlichen Besoldungsausgaben (ca. 31 Mio. $€$ ) im BBesG festgeschrieben wird; d.h. Dienststellen sind zukünftig verpflichtet, das Budget für die Leistungshonorierung vollständig zu nutzen.

\begin{tabular}{|c|c|}
\hline \multicolumn{2}{|c|}{$\begin{array}{l}\text { Übersicht 1: Fiktive Auswirkungen der Leistungselemente in der } \\
\text { Beamtenbesoldung auf das Ruhegehalt }\end{array}$} \\
\hline Beamtin A9 (Bund, ledig, 40 Dienstjahre) & Betrag bei Pensionseintritt 08/2009 \\
\hline Grundgehalt des letzten Amtes (Stufe 8): & $2.877,00 € \mathrm{mtl}$ \\
\hline Max. Leistungszulage* (LZ) 2009: & $154,42 € \mathrm{mtl}$ \\
\hline Max. Leistungsprämie (LP) 2009: & $2.206,00 €$ (einmalig pro Jahr) \\
\hline Ruhegehaltsfähige Dienstbezüge (2009): & $2.877,00 € \mathrm{mtl}$ \\
\hline Abgesenkter Ruhegehaltssatz (2009): & $72,56 \%$ \\
\hline Ruhegehalt (2009): & $2.087,55 € \mathrm{mtl} .(100 \%)$ \\
\hline Fiktives Ruhegehalt, bei max. LZ: & $2.199,60 € \mathrm{mtl} .(105,4 \%))$ \\
\hline Fiktives Ruhegehalt, bei max. LP: & $2.220,94 € \mathrm{mtl} .(106,4 \%)$ \\
\hline Fiktives Ruhegehalt, max. LZ und LP: & $2.332,99 € \mathrm{mtl} .(111,8 \%)$ \\
\hline \multicolumn{2}{|l|}{$\begin{array}{l}\text { * } 7 \% \text { des Anfangsgrundgehalts A } 9 . \\
\text { ** Anfangsgrundgehalt A } 9 .\end{array}$} \\
\hline Quelle: Berechnungen der Autoren. & MITTEILUN \\
\hline
\end{tabular}

Die Forderung, dass sich Leistung auch auf die Beamtenversorgung auswirken soll (Schily et al. 2005; Lorse 2005, S. 445), wurde im DNeuG nicht umgesetzt. In welcher Größenordnung Ruhegehaltssteigerungen durch eine Berücksichtigung der Leistungselemente möglich gewesen wären, wird durch die beispielhafte Vergleichsberechnung in Übersicht 1 zum Ruhegehalt einer Beamtin des gehobenen Dienstes (Grundgehaltstabelle ab 1.7.2009) dargestellt. Insbesondere verdeutlicht das Beispiel jedoch, welche strukturellen Probleme innerhalb der Ruhegehaltsberechnung zu lösen sind, wenn die Leistungskomponenten der Besoldung in der Beamtenversorgung berücksichtigt werden sollen.

Die exemplarische Berechnung in Übersicht 1 weist mögliche Steigerungsraten des monatlichen Versorgungsniveaus um bis $\mathrm{zu} 11,8 \%$ aus, wenn die Leistungszulage und die Leistungsprämie einer Beamtin (Besoldungsgruppe A 9) vollständig auf das Ruhegehalt durchschlagen würden. Potenzielle Ruhegehaltserhöhungen in dieser Größenordnung könnten durchaus Leistungsanreize auf Beamte entfalten und die Leistungsorientierung im öffentlichen Dienst stärken. Gleichwohl verdeutlicht die Ruhegehaltsermittlung, dass bei der derzeitigen Ausgestaltung der Ruhegehaltsformel die Leistungsentgelte „aus dem letzten Amt" direkt in das Ruhegehalt einfließen würden, während Leistungsentgelte der restlichen Lebensarbeitszeit unberücksichtigt bleiben. Somit wäre eine transparente und vom Bezugszeitpunkt unabhängige Berücksichtigung der Leistungselemente nicht gewährleistet. Folglich erschwert die derzeitige Ausgestaltung des Versorgungssystems eine angemessene Berücksichtigung der individuellen Leistung der Beamten bei der Pension. Reformansätze in Richtung einer Beamtenversorgung, die
Leistungsaspekte in der gesamten Erwerbsbiografie berücksichtigt, sollten demzufolge die Problematik umfassend angehen und am Alimentationsgrundsatz der „Versorgung aus dem letzten Amt“ ansetzen.

An dieser Stelle drängt sich die Frage nach den Gründen für den mangelnden Reformeifer des Gesetzgebers auf. Ist die Nichtberücksichtigung der kostenneutral eingeführten Leistungselemente beim $\mathrm{Ru}$ hegehalt den leeren Staatskassen geschuldet? Eine solche Vermutung liegt nahe, da diese Nichtberücksichtigung eine implizite Kürzung der Beamtenversorgung darstellt. Der Perspektivwechsel von der Mikro- zur Makroebene der Ausgabenseite der Beamtenversorgung soll im Folgenden weitere Anhaltspunkte für die Einordnung der Gesetzgebung zum Versorgungsrecht unter „Modernisierung durch Leistungsorientierung“ bzw. unter „Sparzwang aufgrund von Finanzproblemen der öffentlichen Hand" liefern.

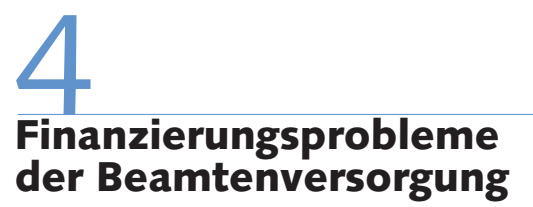

In Deutschland hat neben der gesetzlichen Rentenversicherung gerade die Beamtenversorgung mit großen finanziellen Problemen zu kämpfen (BMI 2005; Färber 1995).

\footnotetext{
Darüber hinaus ergeben sich Auswirkungen auf das verfügbare Einkommen für die private Altersvorsorge. Für Beamte, die keine Leistungszahlungen erhalten, mindert sich durch die Kürzung der Grundbesoldung auch das für die private Vorsorge zur Verfügung stehende Einkommen. Für die Bezieher der Leistungskomponenten fällt der Effekt auf das Einkommen in der aktiven Dienstzeit unterschiedlich aus und richtet sich nach der Höhe der variablen Leistungsbestandteile.
} 


\section{Abb. 1: Versorgungsausgaben der Gebietskörperschaften}

2005-2050 - alternative Berechnungen, in Mrd. Euro -

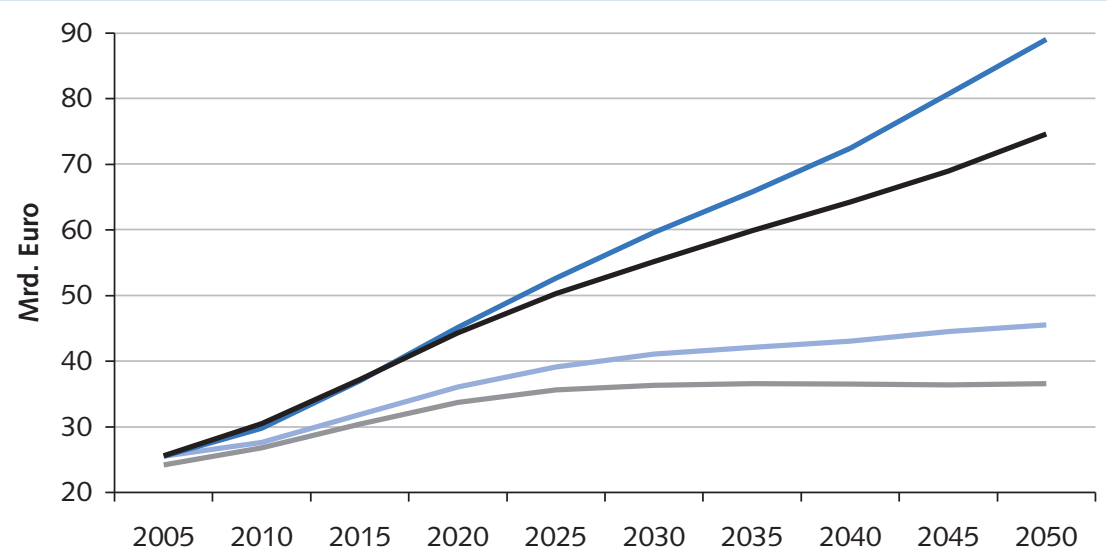

Der doppelte Alterungsprozess belastet das Versorgungssystem der Beamten in besonderem Maße (Besendorfer et al. 2006), da der überwiegende Teil der Pensionsausgaben aus dem laufenden Steueraufkommen finanziert wird. So führen vor allem die Personalexpansion im öffentlichen Dienst seit Ende der 1960er bis Anfang der 1980er Jahre und eine weiter ansteigende Lebenserwartung der Beamten zu höheren Pensionsausgaben. Im Gegenzug belastet die niedrige Geburtenziffer (Ehrentraut/Heidler 2008, S. 232) die Finanzierungsbasis der Beamtenversorgung durch eine abnehmende Zahl von Steuerzahlern.

\subsection{DIE ENTWICKLUNG DER VERSORGUNGSAUSGABEN}

Im Folgenden soll dieser Sachverhalt anhand aktueller Modellrechnungen ${ }^{6}$ (Färber et al. 2009) zur Entwicklung der Versorgungsausgaben von Bund, Ländern und Gemeinden verdeutlicht werden. Die Vorausberechnungen quantifizieren die Versorgungslast der Gebietskörperschaften ausgehend vom Basisjahr 2005 bis zum Jahr 2050 unter Zugrundelegung des derzeit geltenden Rechts. ${ }^{7}$ Die künftigen Versorgungsausgaben hängen dabei von der Veränderung der Versorgungsempfängerzahlen und von der Zunahme der Versorgungsbezüge ab. Hierbei erfolgt die Fort- schreibung der Versorgungsempfängerzahlen ausgehend vom Bestand der aktiven und passiven Beamten im Jahr 2005 unter Zuhilfenahme der Pensionseintrittswahrscheinlichkeiten der Jahre 2001 bis 2005 sowie der Sterbetafel 2003/ 2005, die im Zeitablauf an die Basisannahme zur Lebenserwartung der 11. koordinierten Bevölkerungsvorausberechnung angepasst wird.

Abbildung 1 veranschaulicht die Entwicklung der Versorgungsausgaben bis 2050. Sie wird zunächst durch ein Basisszenario und ein Szenario I dargestellt. Das Basisszenario legt für den gesamten Projektionszeitraum die spezifischen Durchschnittsbezüge des Basisjahres 2005 zugrunde. Diese Berechnungsvariante ohne Anpassung der Versorgungsbezüge zeigt, wie sich die zahlenmäßige und strukturelle Entwicklung der Versorgungsempfänger auf die Versorgungsausgaben auswirkt. Im Basisszenario steigen die Versorgungsausgaben der Gebietskörperschaften von 25,55 Mrd. € im Jahr 2005 bis 2050 um $78 \%$ auf 45,55 Mrd. $€$ an. Dem Szenario I liegt über den gesamten Berechnungszeitraum von 2005 bis 2050 die Annahme zugrunde, dass die Pensionäre jährlich moderate Versorgungsanpassungen in Höhe von 1,5\% erhalten. Die Versorgungsausgaben erhöhen sich hier bis 2050 um $248 \%$ auf 89,04 Mrd. €.

Der starke Anstieg der Pensionsausga- ben erklärt sich aus dem Wachstum der Versorgungsempfängerzahlen. Waren es 2005 bei den Gebietskörperschaften noch 914.600 Versorgungsempfänger, so wird deren Anzahl bis 2050 auf 1,67 Mio. anwachsen. Während der Bund nur mit einem Anstieg von 207.000 im Jahr 2005 auf 237.000 im Jahr 2050 rechnen muss, was nicht zuletzt auf das altersbedingte Ausscheiden der Pensionärsgruppe nach Art. 131 GG zurückzuführen ist, sind die Länder mit einer Verdopplung von 600.000 auf 1,24 Mio. besonders stark betroffen. Auch bei den Gemeinden ist der Anstieg von 107.500 auf 192.500 beträchtlich.

Die offiziellen Vorausberechnungen der Bundesregierung weisen einen deutlich schwächeren Anstieg für Empfängerzahlen und Versorgungsausgaben aus (BMI 2005, S. 333ff.). Dies ist insbesondere auf überholte Annahmen zur künftigen Entwicklung der Lebenserwartung der Versorgungsempfänger zurückzuführen. In dem hier präsentierten Modell steigt die fernere Lebenserwartung der 65-jährigen Beamtinnen bis 2050 auf 25,3 Jahre und die der Beamten auf 21,8 Jahre an. Dies führt in den neuen Modellrechnungen zu längeren Pensionsbezugszeiten als noch vom Dritten Versorgungsbericht 2005 erwartet (Färber et al. 2009, S. 139ff.). Für einen direkten Vergleich der Ergebnisse sind in Abbildung 1 die Berechnungsvarianten aus dem Dritten Versorgungsbericht ${ }^{8}$ (BMI 2005) mit identischen Annahmen hinsichtlich der Entwicklung der Versorgungsbezüge dargestellt.

\subsection{ANSTEIGENDE VERSORGUNGS- STEUERQUOTEN}

Um nun die zu erwartende Haushaltsbelastung durch die Beamtenversorgung darzustellen, werden die vorausberechneten Versorgungsausgaben ins Verhältnis zu den künftigen Steuereinnahmen von Bund, Ländern und Gemeinden gesetzt. Solche Versorgungssteuerquoten stellen die jährlichen Versorgungsausgaben für Pensionäre als Prozentsatz der Steuereinnahmen der

6 Die hier präsentierten Modellrechnungen wurden auf Basis der Ist-Werte des Jahres 2005, die insbesondere die geleisteten Sonderzahlungen berücksichtigen, aktualisiert.

7 Die Modellrechnungen basieren auf Daten des Statistischen Bundesamtes und stellen auf die Rechtslage im Jahr $2008 \mathrm{ab}$.

8 Der Vierte Versorgungsbericht aus dem Jahr 2009 enthält lediglich Vorausberechnungen für den Bund. 
Tabelle 1: Vorausberechnung der Versorgungssteuerquoten der

Gebietskörperschaften - in \% -

\begin{tabular}{|c|c|c|c|c|c|c|c|c|c|c|c|c|}
\hline & \multicolumn{4}{|c|}{ Basisszenario } & \multicolumn{4}{|c|}{ Szenario I } & \multicolumn{4}{|c|}{ Szenario II } \\
\hline & Bund & Länder & $\begin{array}{l}\text { Gemein- } \\
\text { den }\end{array}$ & Gesamt & Bund & Länder & $\begin{array}{l}\text { Gemein- } \\
\text { den }\end{array}$ & Gesamt & Bund & Länder & $\begin{array}{l}\text { Gemein- } \\
\text { den }\end{array}$ & Gesamt \\
\hline 2005 & 2,47 & 9,18 & 4,85 & 5,62 & 2,47 & 9,18 & 4,85 & 5,62 & 2,47 & 9,18 & 4,85 & 5,62 \\
\hline 2025 & 2,56 & 15,55 & 6,88 & 8,63 & 2,10 & 12,72 & 5,63 & 7,06 & 2,55 & 15,47 & 6,85 & 8,59 \\
\hline 2050 & 2,97 & 18,00 & 8,44 & 10,06 & 1,93 & 11,68 & 5,48 & 6,53 & 3,00 & 18,16 & 8,51 & 10,15 \\
\hline
\end{tabular}

entsprechenden Periode dar. Über den Zeitablauf betrachtet, geben die Quoten Auskunft darüber, in welchem Maße die jeweiligen Steuereinnahmen durch die Versorgungsausgaben in Anspruch genommen werden.

Die Vorausberechnung der Versorgungsausgaben wurde hier um ein Szenario II mit jährlichen Versorgungsanpassungen in Höhe von 2,5 \% ergänzt. In diesem Fall würden die Versorgungsausgaben der Gebietskörperschaften bis 2050 um $442 \%$ auf 138,42 Mrd. $€$ ansteigen. Im Basisszenario werden zur Berechnung der Versorgungssteuerquote konstant die Steuereinnahmen aus dem Jahr 2005 zugrunde gelegt. Für die Vorausberechnung der Steuereinnahmen in den Szenarien I und II wird die normierte Steuerquote der letzten zehn Jahre von 21,24 \% und die normierte Verteilung der Steuereinnahmen auf die Gebietskörperschaften bis ins Jahr 2050 fortgeschrieben. Zur langfristigen Bestimmung der gesamtwirtschaftlichen Entwicklung sind die Modellrechnungen mit den Basisannahmen einer makroökonometrischen Vorausberechnung der International Labour Organization (ILO) koordiniert (Scholz 2009). ${ }^{9}$

Tabelle 1 zeigt, dass sich in Zukunft die Haushaltsbelastungen bei Bund, Ländern und Gemeinden unterschiedlich entwickeln werden. Der Bund, dessen Versorgungssteuerquote derzeit mit 2,47 \% recht niedrig ist, muss im Basisszenario nur mit einer geringen Mehrbelastung durch die Versorgungsausgaben rechnen. Im Szenario I, in welchem die Versorgungsempfänger bis 2050 per Annahme (einer jährlichen Inflationsrate von 1,5\%) lediglich einen Inflationsausgleich aus ihren Versorgungsanpassungen erhalten, wird der Bundeshaushalt sogar entlastet. Die Steuereinnahmen steigen in diesem Fall stärker an als die Versorgungsbezüge. Auf die Gemeinden und insbesondere die Länder kommt in den nächsten Dekaden eine Erhöhung der Versorgungssteuerquote zu. Im Szenario II werden die Versorgungsausgaben der Länder 2050 ganze 18,16 \% der Steuereinnahmen beanspruchen. Ein Vergleich der Quoten aus dem Basisszenario mit dem Szenario II zeigt, dass das gesamtwirtschaftliche Wachstum auch bei Bezügeerhöhungen von 2,5\% den Anstieg der Versorgungsausgaben bis 2025 noch leicht abfedern kann. Anschließend sinkt im Modell das nominale Wachstum demografiebedingt auf unter 2,5 \% und führt in Szenario II zu einem stärkeren Anstieg der Versorgungssteuerquote als im Basisszenario.

Insgesamt wird die Versorgungssteuerquote der gesamten Gebietskörperschaften in allen Szenarien ansteigen. Diese Entwicklung zeigt, dass die geforderte Nachhaltigkeit im Finanzierungssystem der Beamtenversorgung (Karl-Bräuer-Institut 2006) bisher nicht erreicht wurde. Vielmehr kommen auf die Gebietskörperschaften durch die Versorgungslasten große Finanzierungsprobleme zu.

\section{Modernisierung und Sparzwang}

\subsection{DIE REFORMEN}

Vor dem Hintergrund steigender Versorgungslasten der Beamtenversorgung, die einen erheblichen Teil der Tragfähigkeitslücke (SVR 2003) in Deutschland verursachen, hat der Gesetzgeber bereits vor dem DNeuG eine Reihe von Reformmaßnahmen veranlasst. Diese Reformprozesse setzen seit 20 Jahren vor allem bei der Höhe der Versorgungsleistungen (Färber et al. 2009, S. 135ff.), und nicht bei den Strukturprinzipien oder Berechnungsgrundsätzen der Beamtenversorgung an. Unter anderem wird das Versorgungsniveau selbst seit 2003 schrittweise reduziert. Darüber hinaus wirkt der Gesetzgeber dem Frühpensionierungstrend mit Versorgungsab- schlägen entgegen. Außerdem werden seit 1999 die Versorgungsanpassungen gemindert, um Versorgungsrücklagen zu bilden. ${ }^{10}$ Die bedeutendsten Reformmaßnahmen der letzten zwei Dekaden zeigt Übersicht 2.

Im Rahmen der Änderungen sind in der Regel die Kosten senkenden Reformmaßnahmen aus der gesetzlichen Rentenversicherung „wirkungsgleich“ auf die Beamtenversorgung übertragen worden (BMI 2005, S. 86; Bull 2008, S. 30; Ruland 2002). Allerdings verdeutlichen die Vorausberechnungen der Versorgungsausgaben, dass durch diese Maßnahmen die öffentlichen Haushalte nach wie vor nicht nachhaltig entlastet werden. Obwohl bei der Projektionsrechnung die bis 2008 beschlossenen Gesetzesänderungen berücksichtigt werden, steigen die Versorgungslasten weiter an. Die Zahlen weisen darauf hin, dass den Beamten in den nächsten Jahren weitere Änderungen hinsichtlich ihrer Altersversorgung bevorstehen und dass beim Versorgungsniveau erneut nachkorrigiert werden wird. Es stellt sich daher die Frage, ob nicht vielmehr eine grundlegende Neuausrichtung des Alterssicherungssystems auf individuelle Leistung der Bediensteten und auf Nachhaltigkeit notwendig ist.

\subsection{DISKUSSION}

Die erfolgten Reformen in Form von Kürzungen des Versorgungsniveaus in der Beamtenversorgung lassen sich mit Blick auf die Vorausberechnungen aus Abschnitt 4 als Reaktion auf die steigenden Versorgungslasten und die allgemeine Haushalts-

\footnotetext{
9 Für weitere Informationen zu den Parametern und Annahmen der Modellrechnungen vgl. Färber et al. 2009.

10 Distributive Aspekte werden in dem vorliegenden Beitrag ausgeklammert. Weitere Forschungsarbeiten könnten an dieser Stelle beispielsweise die Auswirkungen der Reformen auf das Versorgungsniveau verschiedener Beamtentypen und -kohorten analysieren.
} 


\begin{tabular}{|c|c|}
\hline \multicolumn{2}{|c|}{ Übersicht 2: Reformen der Beamtenversorgung seit 1989} \\
\hline Reformgesetz/Jahr & Änderungen der Versorgungsleistungen \\
\hline \multirow{3}{*}{$\begin{array}{l}\text { Beamtenversorgungs- } \\
\text { änderungsgesetz } 1989 \\
\text { Geltung ab 1.1.1992 }\end{array}$} & $\begin{array}{l}\text { Abschaffung der degressiven Ruhegehaltsstaffel und Einführung } \\
\text { der linearen Ruhegehaltsskala }\end{array}$ \\
\hline & $\begin{array}{l}\text { Anhebung der Dienstjahre zur Erlangung der Höchstversorgung } \\
\text { i. H.v. } 75 \% \text { von } 35 \text { auf } 40 \text { Jahre }\end{array}$ \\
\hline & $\begin{array}{l}\text { Einführung eines Versorgungsabschlags i. H.v. 3,6 \% für jedes Jahr } \\
\text { vor Vollendung des 65. Lebensjahres (bei Inanspruchnahme der } \\
\text { Antragsaltersgrenze) }\end{array}$ \\
\hline \multirow{3}{*}{$\begin{array}{l}\text { Dienstrechtsreformgesetz } 1997 \\
\text { Geltung ab 1.7.1997 }\end{array}$} & $\begin{array}{l}\text { Anhebung der allgemeinen Antragsaltersgrenze für den vorzeitigen } \\
\text { Ruhestand von } 62 \text { auf } 63 \text { Jahre }\end{array}$ \\
\hline & $\begin{array}{l}\text { Schrittweises Vorziehen der Einführung des Versorgungsabschlags } \\
(0,6 \% 1998 \text { bis 3,6\% ab 2003) }\end{array}$ \\
\hline & Kürzung ruhegehaltsfähiger Ausbildungszeiten auf 3 Jahre \\
\hline \multirow{2}{*}{$\begin{array}{l}\text { Versorgungsreformgesetz } 1998 \\
\text { Geltung ab 1.7.1998 }\end{array}$} & $\begin{array}{l}\text { Einführung und Bildung einer Versorgungsrücklage durch } \\
\text { schrittweise Absenkung des Besoldungs- und Versorgungsniveaus } \\
\text { um } 3 \% \text { (15 mal 0,2 Prozentpunkte in den Jahren } 1999 \text { bis 2013) }\end{array}$ \\
\hline & $\begin{array}{l}\text { Berücksichtigung der Stellenzulagen als ruhegehaltsfähige Dienst- } \\
\text { bezüge entfällt; Verschärfung der Hinzuverdienstregelungen }\end{array}$ \\
\hline \multirow{5}{*}{$\begin{array}{l}\text { Versorgungsänderungsgesetz } \\
2001 \\
\text { Geltung ab 1.1.2003 }\end{array}$} & Absenkung des Versorgungsniveaus in 8 Schritten: \\
\hline & $\begin{array}{l}\text { Höchstruhegehaltssatz von } 75 \% \text { auf } 71,75 \% \text { der ruhegehalts- } \\
\text { fähigen Dienstbezüge }\end{array}$ \\
\hline & $\begin{array}{l}\text { dementsprechende Absenkung des jährlichen Steigerungssatzes } \\
\text { von } 1,875 \% \text { auf } 1,79375 \%\end{array}$ \\
\hline & $\begin{array}{l}\text { Die Anpassungsminderungen um 0,2 Prozentpunkte (s. o.) werden } \\
\text { ausgesetzt und erst im Anschluss an die } 8 \text {. Anpassung wieder } \\
\text { aufgenommen }\end{array}$ \\
\hline & $\begin{array}{l}\text { Absenkung des Witwen- bzw. Witwergeldes von } 60 \% \text { auf } 55 \% \\
\text { der zugrunde liegenden Beamtenpension }\end{array}$ \\
\hline \multirow{3}{*}{$\begin{array}{l}\text { Dienstrechtsneuordnungsgesetz } \\
2009 \\
\text { Geltung ab 11.2.2009 }\end{array}$} & Schrittweise Anhebung des Pensionseintrittsalters auf 67 Jahre \\
\hline & $\begin{array}{l}\text { Schrittweise Ausweitung des max. Versorgungsabschlages auf } \\
14,4 \% \text { (Festhalten der Antragsaltersgrenze bei } 63 \text { ) }\end{array}$ \\
\hline & $\begin{array}{l}\text { Abschlagsfreier Pensionseintritt bei } 45 \text { Jahren ruhegehaltsfähiger } \\
\text { Dienstzeiten }\end{array}$ \\
\hline
\end{tabular}

lage der Gebietskörperschaften interpretieren. Somit sind die bisherigen Reformen eher Ausdruck eines öffentlichen Sparzwangs und weniger Teil eines ausdifferenzierten Modernisierungskonzepts für den öffentlichen Dienst. Der Gesetzgeber hat trotz höchstrichterlicher Betonung (BVerfG, Urt. v. 27.9.2005 - 2 BvR 1387/02, Rn. 131f.) der strukturellen Unterschiede der Beamtenversorgung und der gesetzlichen Rentenversicherung (GRV) bereits seit 1992 eine Konvergenz der beiden Alterssicherungssysteme vorangetrieben ( $\mathrm{Ru}$ land 2002, S. 948). An dieser Stelle sei deshalb auf die Bifunktionalität der Beamtenversorgung, die anders als die GRV neben der ersten Säule der Alterssicherung zusätzlich die betriebliche Alterssicherung (zweite Säule) abdeckt (BMGS 2003, S. 123), verwiesen. Bei der Diskussion um die Grenzen weiterer Kürzungen sollte neben dem aus Art. 33 Abs. 5 GG abgeleiteten Alimentationsprinzip (Lindner 2007, S. 223) stets der bifunktionale Charakter der Beamtenversorgung berücksichtigt bleiben. ${ }^{11}$

Auch die Einführung leistungsorientierter Besoldungselemente hat implizit zu

mission oder des Eckpunktepapiers „Neuc Wege im öffentlichen Dienst" (Schily et al. 2005), die Lösungsansätze hin zu einem umfassenden Modell einer leistungsorientierten Besoldung und Versorgung enthielten, wurden vom DNeuG 2009 nicht berücksichtigt. Im System der Beamtenversorgung macht sich zusätzliche Leistung somit nicht bezahlt.

Eine Einbeziehung der Leistungskomponenten in die Versorgung und somit die Einkehr von leistungsbezogenen Elementen in das Alterssicherungssystem der Beamten wäre jedoch wünschenswert und sollte nicht dem Sparzwang zum Opfer fallen. Ganzheitliche Lösungen, die eine Stimmigkeit zwischen einem leistungsorientierten Bezahlungssystem und dem organisationalen Kontext des öffentlichen Dienstes schaffen (Jörges-Süß 2006, S. 40), erfordern inhaltlich aufeinander abge- stimmte Reformen der Beamtenbesoldung und -versorgung.

Nachdem in Art. 33 Abs. 5 GG explizit festgeschrieben wurde, dass das Beamtenrecht auch „fortzuentwickeln“ ist, scheint die „Reformbremse“ Alimentationsprinzip (Bull 2006, S. 244ff.) einer Leistungsausrichtung der Beamtenversorgung nicht mehr im Weg zu stehen. Vielmehr lässt es selbst das tradierte Alimentationsprinzip $\mathrm{zu}$, dass bei der Versorgung der Beamten zumindest teilweise auf die zuvor erbrachte Leistung abgestellt wird. Vor diesem Hintergrund ist die Einführung eines "Mischsystems“ aus Basisversorgung und einem variablen leistungsbezogenen Versorgungsanteil möglich, wenn die Basisversorgung eine ämterbezogene Differenzierung beibehält und einen Kernbestand der Alimentation unangetastet lässt (Lorse 2005, S. 454). Allerdings haben die Ausführungen zur Ruhegehaltsberechnung in Abschnitt 3 verdeutlicht, dass die derzeitigen Strukturprinzipien der Beamtenversorgung eine konsequente zeitanteilige Berücksichtigung der leistungsorientierten Besoldungskomponenten beim Ruhegehalt erschweren. Die Umsetzung des Alimentationsprinzips als Versorgung aus dem letzten Amt ist für nur phasenweise in der Erwerbsbiografie gewährte Leistungselemente nicht anwendbar. Denkbar wären für diesen variablen Leistungsanteil nach der Bezugsdauer gewichtete, zusätzliche Versorgungsbezüge, die wie alle Versorgungsansprüche periodengerecht bereits bei der Entstehung (in der aktiven Erwerbsphase) in den entsprechenden Haushalt eingestellt werden sollten.

\section{0 \\ Ausblick}

Aus der Perspektive des öffentlichen Dienstes als Arbeitgeber haben die fortlaufenden Absenkungen des Versorgungsniveaus und eine ausbleibende Berücksichtigung leistungsorientierter Besoldungselemente bei der Versorgung weitreichende Konsequenzen. Vor dem Hintergrund des demografischen Wandels wird der öffentliche Dienst

11 Zusätzlich sind die Wechselwirkungen der Besoldungs- und Versorgungsreformen mit der Möglichkeit und Notwendigkeit der privaten, eigenverantwortlichen Altersvorsorge (dritte Säule) zu beachten. 
als Arbeitgeber künftig zunehmend mit der Privatwirtschaft in Konkurrenz um qualifizierte Nachwuchs- und Fachkräfte treten, da tendenziell weniger Bewerber zur Verfügung stehen werden (Bull 2008, S. 228f). Kürzungen bei den Versorgungsbezügen sind in diesem Wettbewerb um Leistungsträger ebenso nachteilig (Färber 1998, S. 979) wie die Abkopplung der Beamtenbesoldung von der allgemeinen Reallohnentwicklung in Deutschland (Sinn 2004). An dieser Stelle sollte der bis dato die Versorgungsreformen dominierende Spar- zwang zumindest um eine Harmonisierung der Leistungsausrichtung in Besoldung und Versorgung ergänzt werden. Eine diesbezügliche Einheitlichkeit von Besoldung und Versorgung würde zur leistungsorientierten Umgestaltung des öffentlichen Dienstes beitragen und seine Attraktivität als Arbeitgeber erhöhen.

Um die Leistungskomponenten bei der Alterssicherung der Beamten einzubeziehen und um fortlaufenden Kürzungen beim Versorgungsniveau entgegenzuwirken, ist jedoch eine grundlegende Neuge- staltung des Systems nötig. Eine Fortentwicklung des Alimentationsprinzips erscheint notwendig, zumal bereits nach heutigem Rechtsverständnis teilweise auf die individuelle Leistung der Beamten abgestellt werden darf. Ob vor diesem Hintergrund eine (partielle) Umstellung der Ruhegehaltsberechnung auf ein Versorgungspunktemodell möglich ist, welches die leistungsbezogenen Besoldungselemente der gesamten Dienstzeit und die Bifunktionalität der Beamtenversorgung berücksichtigt, sollte geprüft werden.

\section{LITERATUR}

Battis, U. (2005): Zum Entwurf eines Gesetzes zur Reform der Strukturen des öffentlichen Dienstrechts, in: Zeitschrift für Beamtenrecht 10, S. 325329

Becker, J./Algermissen, L./Falk, T. (2007): Prozessorientierte Verwaltungsmodernisierung. Prozessmanagement im Zeitalter von E-Government und New Public Management, Berlin

Besendorfer, D./Dang, E. P./Raffelhüschen, B. (2006): Schulden und Versorgungsverpflichtungen der Länder. Was ist und was kommt?, in: Wirtschaftsdienst 9, S. 572-579

Biermann, E./Briedrigkeit, M./Kammradt, N./Raab, S./Schlenzka, M. (2005): Ratgeber Beamtenrecht. Laufbahnrecht und Qualifizierung/ Besoldung/Arbeitszeit und Urlaub/Reise und Umzugskosten/Beamtenversorgung/Beihilfe, Frankfurt am Main

Bolay, F. (2007): Leistungsbewertung oder "Nasenprinzip"? Kurswechsel bei der Weiterentwicklung der leistungsorientierten Vergütung für Beamte; in: Verwaltung und Management 2, S. 104-109

Bundesministerium des Inneren (BMI) (2005): Dritter Versorgungsbericht der Bundesregierung, Berlin

Bundesministerium für Gesundheit und Soziale Sicherung (BMGS)

(2003): Nachhaltigkeit in der Finanzierung der sozialen Sicherungssysteme. Bericht der Kommission, Berlin

Bull, H. P. (2006): Bürokratieabbau und Dienstrechtsreform, in: Die Öffentliche Verwaltung 6, S. 241-249

Bull, H. P. (2008): Politische Verantwortung für einen leistungsstarken öffentlichen Dienst, in: Verwaltung und Management 5, S. 227-234 Bull-Kommission (2003): Bericht der von der Landesregierung Nordrhein-Westfalen eingesetzten Kommission Zukunft des öffentlichen Dienstes - öffentlicher Dienst der Zukunft, Düsseldorf

Drescher, A. (2007): Dienstrechtsreformen in Bund und Ländern. Auswirkungen auf Status, Besoldung, Versorgung und Wettbewerbsfähigkeit. 2. Bonner Dienstrechtssymposion, in: Recht im Amt 5, S. 261-266
Ehrentraut, O./Heidler, M. (2008): Demografisches Risiko für die Staatsfinanzen? Koordinierte Bevölkerungsvorausberechnungen im Vergleich, in: Sozialer Fortschritt 9, S. 231-241

Färber, G. (1995): Revision der Personalausgabenprojektion der Gebietskörperschaften bis 2030 unter Berücksichtigung neuerer Bevölkerungsvorausschätzungen, der deutschen Einigung und der Beamtenversorgungsreform, Speyerer Forschungsberichte 110, Speyer

Färber, G. (1998): Mittel- und langfristige Entwicklung der Beamtenversorgung, in: Cramer, J.-E./Förster, W./Ruland, F. (Hrsg.): Handbuch zur Altersversorgung. Gesetzliche, betriebliche und private Vorsorge in Deutschland, Frankfurt am Main, S. 973-994

Färber, G./Funke, M./Walther, S. (2009): Die Entwicklung der Beamtenversorgung in Deutschland seit 1992 und künftige Finanzierungsprobleme der Gebietskörperschaften, in: Die Öffentliche Verwaltung 4, S. 133146

Göser, H./Schlatmann, A. (1998): Leistungsbezahlung in der Besoldung, München

Jörges-Süß, K. (2006): Zahlt sich Leistung aus? Leistungsabhängige Vergütung im öffentlichen Dienst, in: Personalführung 7, S. 34-40

Karl-Bräuer-Institut des Bundes der Steuerzahler (Hrsg.) (2006): Ausgaben für Beamtenpensionen eindämmen. Versorgungsrecht auf Nachhaltigkeit ausrichten, Stellungnahmen 30, Berlin

Lindner, J. F. (2007): Das Alimentationsprinzip und seine offenen Flanken, in: Zeitschrift für Beamtenrecht 7-8, S. 222-230

Lorse, J. (2005): Eckpunktepapier "Neue Wege im Dienstrecht": Wie verbindet man Eckpunkte zu Grundlinien einer Reform?, in: Die Öffentliche Verwaltung 11, S. 445-457

Lorse, J. (2007): Aktuelle tarif- und dienstrechtliche Reformüberlegungen im öffentlichen Dienst - eine Zwischenbilanz, in: Zeitschrift für Beamtenrecht 1-2, S. 24-35 
Mühlenkamp, H. (2008): Leistungsbezahlung im öffentlichen Sektor unter dem Regime der "Kostenneutralität": Warum sie nicht wirklich funktionieren kann. Eine Analyse mit Hilfe der Prinzipal-Agent-Theorie, in: Magiera, S./Sommermann, K.-P./Ziller, J. (Hrsg.): Verwaltungswissenschaft und Verwaltungspraxis in nationaler und transnationaler Perspektive. Festschrift für Heinrich Siedentopf zum 70. Geburtstag, Berlin,

S. 637-653

Murmann, T. (1991): Grundlagen des Beamtenversorgungsrechts, in: Recht im Amt 5, S. 231-246

Ruland, F. (2002): Noch einmal davon gekommen - Zur Reform der Beamtenversorgung, in: Neue Juristische Wochenzeitschrift 13, S. 948949
Sachverständigenrat zur Begutachtung der Gesamtwirtschaftlichen Entwicklung (SVR) (2003): Tragfähigkeitslücke der öffentlichen Haushalte. Auszug aus dem Jahresgutachten 2003/04, in: Staatsfinanzen konsolidieren - Steuersystem reformieren. Jahresgutachten 2003/2004, Ziffern 438-454 und 765-788

Schily, O./Heesen, P./Bsirske, F. (2005): „Neue Wege im öffentlichen Dienstrecht“. Eckpunkte für eine Reform des Beamtenrechts, in: Zeitschrift für Beamtenrecht 7-8, S. 217-221

Scholz, W. (2009): The Social Budget of Germany. Keeping the Welfare State in Perspective, Berlin

Schwahn, F. (2007): Entwicklungen im öffentlich-rechtlichen Alterssicherungssystem, in: Wirtschaft und Statistik 4, S. 395-403

Sinn, H.-W. (2004): Sieben Wahrheiten über Beamte, ifo Standpunkt 56 